\title{
Synchronous occurrence of mature cystic teratoma of the fallopian tube and ovary: A case report
}

\author{
JONGCHUL BAEK \\ Department of Obstetrics and Gynecology, Gyeongsang National University Hospital, \\ Changwon, Gyeongsangnam 641-560, Republic of Korea \\ Received April 5, 2015; Accepted May 19, 2016
}

DOI: $10.3892 / \mathrm{ol} .2016 .4747$

\begin{abstract}
Primary carcinoma of the fallopian tube represents $<1 \%$ of all gynecological tumors, and benign tumors of the fallopian tube are encountered even less frequently. Mature teratomas of the fallopian tube and ovary are extremely rare, and to date, only a few cases have been reported. A 31-year-old woman presented with a left adnexal mass that was identified by pelvic ultrasonography during a regular checkup. The patient underwent laparoscopic left salpingectomy and left ovarian cystectomy. Histopathological examination of the removed tissue revealed features compatible with mature cystic teratomas of both the left fallopian tube and ovary. At 26 months post-surgery, the patient underwent a cesarean section at $39+2$ weeks of gestation. A right ovarian cystic mass was incidentally identified during the procedure. Right ovarian cystectomy was performed, and histopathological analysis revealed the mass to be a teratoma. The patient continued to receive annual follow-up after surgical intervention and demonstrated no evidence of disease at a routine 2-year follow-up examination. The discussion of the present case is followed by a brief review of the literature.
\end{abstract}

\section{Introduction}

The defining feature of a mature cystic teratoma is the presence of differentiated components of any combination of two or three embryonal layers (endoderm, mesoderm and ectoderm) (1).

Although mature teratomas are the most frequently occurring ovarian germ cell tumors (16-20\% of all ovarian neoplasms), the incidence of fallopian tube teratomas is extremely low (2), with only $\sim 70$ cases of mature fallopian tube teratoma reported in the literature (2). Teratomas of

Correspondence to: Professor Jongchul Baek, Department of Obstetrics and Gynecology, Gyeongsang National University Hospital, 45 Changidaero, Uichan-gu, Changwon, Gyeongsangnam 641-560, Republic of Korea

E-mail: gmfather@gmail.com

Key words: teratomas, fallopian tube, ovary the fallopian tube are generally solitary tumors. However, in certain cases, they may be accompanied by various ovarian neoplasms. In the literature, the accompanying neoplasms have consisted of ovarian teratomas (including a struma ovarii) in 6 cases, benign ovarian tumors other than teratomas in 3 cases and ovarian cystadenocarcinomas in 2 cases (3-5). Fallopian tube teratomas are usually asymptomatic, but they may occasionally lead to reduced parity, leukorrhea, menstrual irregularity, postmenopausal bleeding or abdominal pain (5). The present study reports the case of patient with a mature cystic teratoma of the left fallopian tube coexisting with mature cystic teratomas of the bilateral ovaries.

\section{Case report}

A 31-year-old woman (gravida 0, para 0) was referred to the Department of Gynecology, Gyeongsang National University Hospital (Gyeongsangnam, Korea) after an adnexal mass was identified during pelvic ultrasonography, which was performed at a regular checkup in April 2012. Computed tomography scans revealed a multi-lobulated, $4.5 \times 3.5-\mathrm{cm}$ cystic mass, with fat and calcification in the left adnexa, which was suspected to be a cystic teratoma of the left ovary. The results of a complete blood count, urinalysis, serological tests, electrocardiogram and chest X-ray were within the normal ranges. Serum cancer antigen (CA)-125 and CA19-9 levels were 18 and $4 \mathrm{U} / \mathrm{ml}$, respectively (normal ranges, $<35$ and $<37 \mathrm{U} / \mathrm{ml}$, respectively).

The patient underwent laparoscopic surgery with a diagnosis of a cystic teratoma of the left ovary. At the time of surgery, the left fallopian tube appeared to be distended by $4 \times 3 \mathrm{~cm}$ and contained cream-colored sebaceous material and hair (Fig. 1A). The ipsilateral ovary also contained cream-colored sebaceous material and hair (Fig. 1B). The uterus, the right ovary and the right fallopian tube were evaluated as normal. The patient subsequently underwent a left salpingectomy and left ovarian cystectomy.

The CT images were retrospectively evaluated with focus on the left fallopian tube components after surgery. Computed tomography scans showed a cystic mass with a fat attenuation component in the left hemipelvis. The mass was connected with a dilated folding tubular structure. The tubular structure was $5 \mathrm{~mm}$ in diameter and demonstrated fat attenuation (-105 HU). These CT findings were consistent with those of fallopian tube teratomas (Fig. 2). 
A

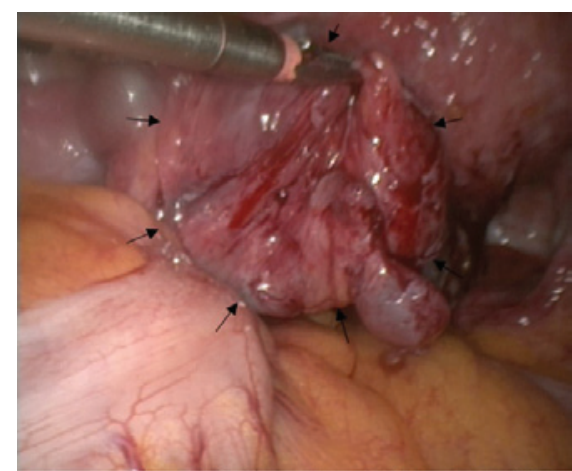

B

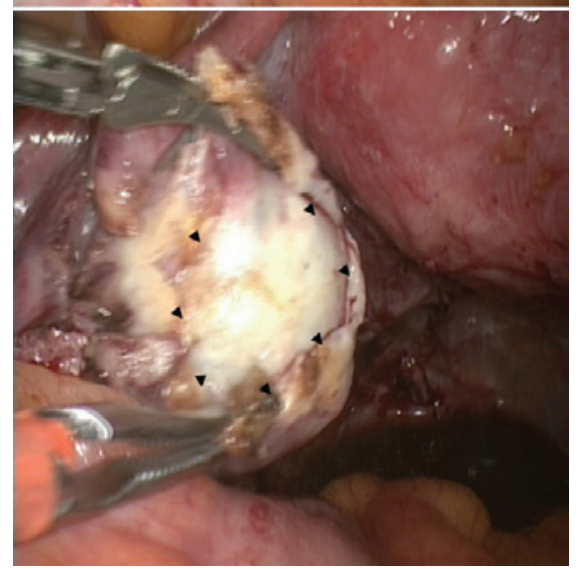

Figure 1. Surgical findings showing (A) a mass containing sebaceous material identified within the ampullary region of the left fallopian tube (black arrows) and (B) an ovarian cyst containing yellow waxy material (black arrowheads).

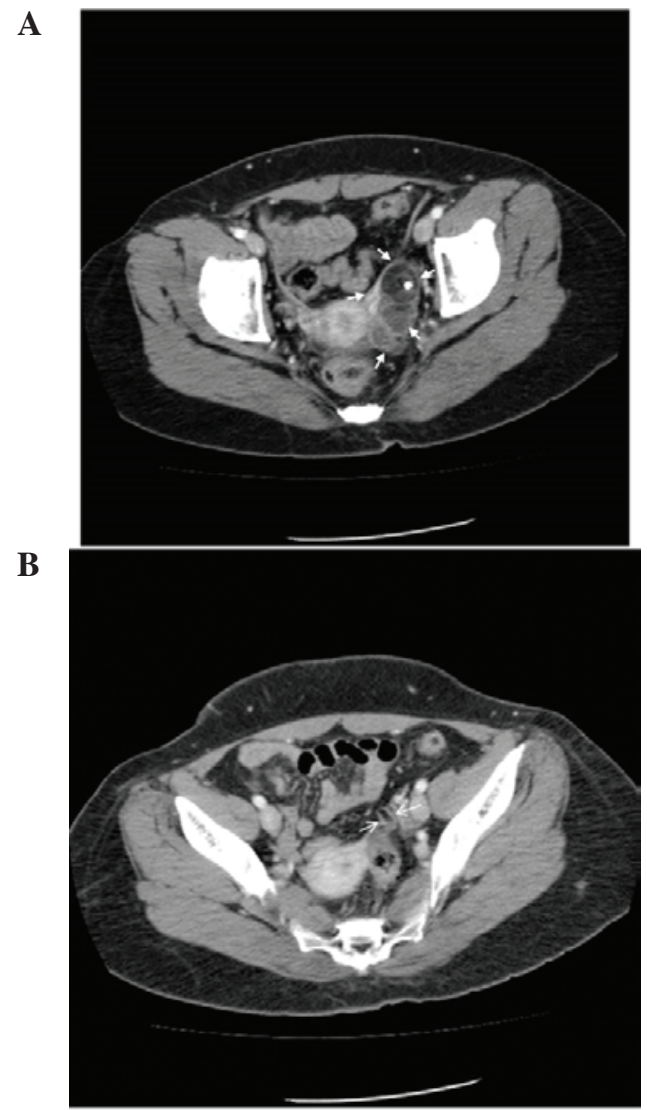

Figure 2. Computed tomography scan showing (A) a 4.5x3.5-cm cystic and fatty mass, with calcification in the left hemipelvis (white arrows). (B) Left tubular structure (white arrows) showing fat attenuation $(-105 \mathrm{HU})$.
A

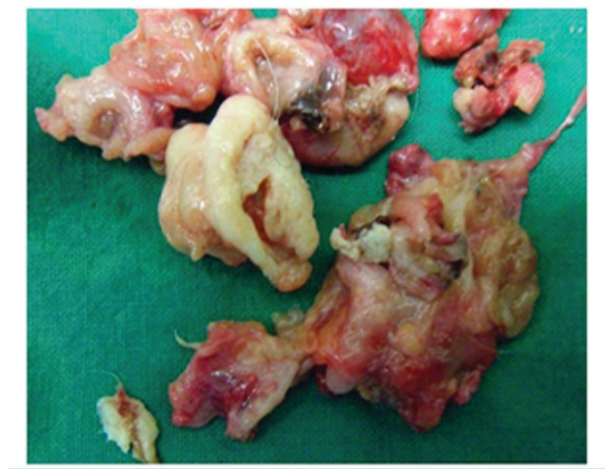

B

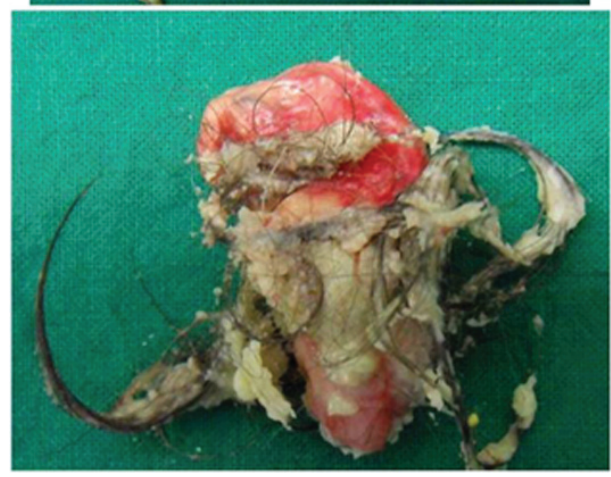

Figure 3. Gross findings of (A) the fallopian tube showing a solid and cystic content. The solid portion contained a plug of grease material and hairs. (B) The ovarian cyst contained hair and fat components.

A

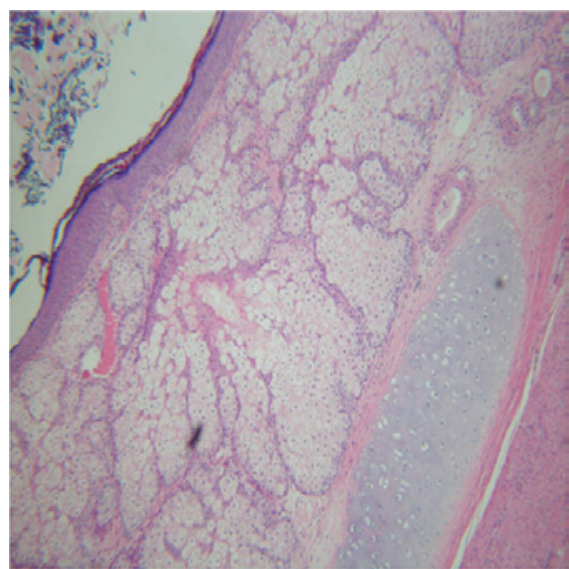

B

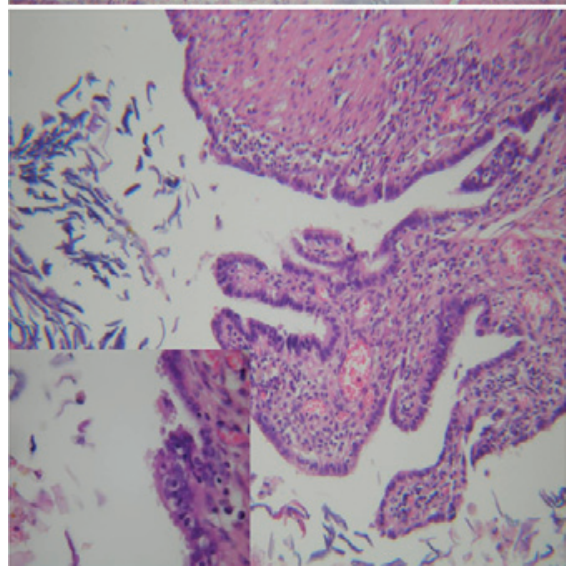

Figure 4. (A) Microscopically, the lining epithelium consisted of well-differentated keratin-producing squamous epithelium with underlying sebaceous glands and mature cartilage (H\&E; magnification, $x 40)$. (B) The fallopian tube contained keratinous debris and exhibited mucosal folds of epithelium (H\&E; magnification, x100). Inset image shows ciliated and non-ciliated fallopian epithelium (H\&E; magnification, $x 400)$. H\&E, hematoxylin and eosin. 
Grossly, a cystic mass, $3.5 \mathrm{~cm}$ in diameter, was present within the ampulla of the fallopian tube, which was filled with fatty tissue and yellowish greasy material and hairs (Fig. 3A). The left ovary exhibited fatty, tan-pink, focally calcified cut surfaces, which were surrounded by a $2.0 \times 2.0 \times 1.5-\mathrm{cm}$ uniloculated cyst containing coarse black hair (Fig. 3B). The samples were grossly examined, dissected, embedded in paraffin blocks and cut into $3 \mu \mathrm{m}$ sections. The sections were then stained using standard hematoxylin and eosin, and representative blocks were selected from each tumor for microscopic analysis. Microscopic examination revealed squamous epithelium with sebaceous glands, and hair follicles with mucous glands and mature cartilage (Fig. 4).

Histopathological examination showed components from each germ cell layer; therefore, the diagnosis of mature cystic teratomas of the left fallopian tube and ovary was confirmed. The patient was discharged from the hospital at 3 days post-surgery. There were no abnormal findings when the patient revisited the Department of Gynecology, Gyeongsang National University Hospital at 10 days post-surgery. At 26 months post-surgery, the patient became pregnant and underwent a cesarean section at $39+2$ weeks due to fetal distress. A cystic mass of the right ovary was incidentally found during cesarean section. A right ovarian cystectomy was performed and histopathology revealed it to be a teratoma. The patient continued to receive annual follow-up after the second surgical intervention and demonstrated no evidence of disease at a routine 2-year follow-up examination. Written informed consent was obtained from the patient for the publication of this report and any accompanying images.

\section{Discussion}

Tumors of the fallopian tube are uncommon. Primary malignant tumors of the fallopian tube account for $0.3-1.9 \%$ of gynecological malignancies (6). Benign tumors of the fallopian tube are even less common, the majority being adenomatoid tumors (7). Teratomas of the fallopian tube are rare, and to date, only $\sim 70$ cases have been reported in the global literature (3). Due to their rarity, the actual incidence and histogenesis of mature cystic teratoma of the fallopian tube remains unknown (8).

This condition affects patients aged between 17-67 years, although the majority of patients are 30-49 years of age (2). An analogous age-associated trend is found for size (range, $0.2-31 \mathrm{~cm}$ in diameter) and weight $(\leq 2,400 \mathrm{~g})(9)$. As a result, the volume of the teratoma, and thus its speed of growth, appears to be inversely proportional to patient age, with juveniles exhibiting greater volumes than older patients. The gravidity of patients with teratomas of the fallopian tube is variable. The majority of studies have reported a high prevalence rate in multipara patients compared with nullipara or primipara patients $(2,3,8)$. The patient in the present case was nullipara. The patient underwent a left ovarian cystectomy and left salpingectomy. At 26 months post-surgery, the patient became pregnant and underwent a cesarean section. A right ovarian teratoma was found incidentally.

The literature suggests that teratomas are slightly more prevalent in the right fallopian tube than in the left; in a recent study, 34 cases occurred on the right side, 28 occurred on the left side and 6 occurred bilaterally (3). Intraluminal teratomas are the most common type and may also occur at intramural and serosal locations (internal layer, 22 cases; serosal layer, 4 cases; muscular layer, 2 cases; and combined, 3 cases) $(3,5)$.

Teratoma is able to develop in any region of the fallopian tube, but the ampulla and isthmus are the most common sites (9). The diagnosis and clinical manifestation of teratomas are associated with their location. Furthermore, teratomas of the fallopian tube have been associated with subfertility, infertility, irregular menstruation, menorrhagia, leukorrhea, ectopic pregnancy, adnexal masses and lower abdominal pain (8-12). The patient in the present study experienced no specific symptoms. Occasionally, tubal teratomas may cause infertility, however, the patient in the present study conceived spontaneously at 26 months post-surgery.

Teratomas of the fallopian tube are generally solitary tumors (2,9-11). However, certain cases have been accompanied by various gynecological neoplasms, including struma ovarii (4), ovarian teratoma (5), uterine myoma $(3,5)$, ectopic pregnancy (8) and uterine anomaly (13) Moreover, certain cases have also been associated with gynecological malignancies, such as ovarian adenocarcinoma (5), endometrial carcinoma (14) and cervical squamous carcinoma (5).

The methods used for the detection of this neoplasm include ultrasonography, pelvic CT scan, nuclear magnetic resonance imaging, hysterosalpingography and laparoscopy $(10,13,15)$. However, tubal teratomas are not usually preoperatively diagnosed due to their ambiguous clinical manifestation. To date, no cases involving preoperative diagnosis have been reported. All reported cases have been identified incidentally during surgical procedures and subsequently confirmed by histopathological analysis. The prognosis for such patients is favorable.

In conclusion, the present case described a patient with a mature cystic teratoma of the left fallopian tube, in addition to bilateral ovarian teratomas. If a patient presents with an adnexal mass, it is important for gynecological surgeons to consider the occurrence of tubal teratomas when making a differential diagnosis. Although the incidence of tubal teratomas is low, it is necessary to be aware of this possibility. It is also important to consider that tubal teratomas may be associated with other gynecological conditions. Thus, the abdomen and pelvis should be inspected in a meticulous manner and every effort should be made to identify coexisting lesions.

\section{References}

1. Robert JK: Mature cystic teratoma. In: Blaustein's Pathology of the Female Genital Tract. 4th edition. Springer-Verlag, New York, pp879-883, 1994.

2. Khatib G, Guzel AB, Kucukgoz-Gulec U, Vardar MA, Musaev A and Melekoglu R: Mature cystic teratoma of the fallopian tube. J Obstet Gynaecol 33: 120-124, 2013.

3. Chao T, Chao J, Kuan LJ, Li YT, Kuo TC, Chang YC and Nieh S: Mature solid teratoma of the fallopian tube associated with uterine leiomyomas. J Chin Med Assoc 71: 425-427, 2008.

4. Hoda SA and Huvos AG: Struma salpingis associated with struma ovarii. Am J Surg Pathol 17: 1187-1189, 1993.

5. Mazzarella P, Okagaki T and Richart RM: Teratoma of the uterine tube. A case report and review of the literature. Obstet Gynecol 39: 381-388, 1972.

6. Raju KS, Barker GH and Wiltshaw E: Primary carcinoma of the fallopian tube. Report of 22 cases. Br J Obstet Gynaecol 88: 1124-1129, 1981. 
7. Fox $\mathrm{H}$ and Buckley $\mathrm{CH}$ : The female genital tract and ovaries. In: Oxford Textbook of Pathology. Volume 2a. McGee JOD, Isaacson PG and Wright NA (eds), pp1613-1614, 1992.

8. Kutteh WH and Albert T: Mature cystic teratoma of the fallopian tube associated with an ectopic pregnancy. Obstet Gynecol 78 984-986, 1991.

9. Ingec M, Kadanali S and Erdogan F: Huge teratoma of the fallopian tube: A case report. J Reprod Med 52: 247-249, 2007.

10. Fujiwara S, Yamashita Y, Yoshida Y, Terai Y, Okuda K and Ohmichi M: Mature cystic teratoma of the fallopian tube. Fertil Steril 94: 2708-2709, 2010.

11. Johnson C and Hansen KA: Mature cystic teratoma of the fallopian tube. Fertil Steril 86: 995-996, 2006.
12. Matts SJ: An unusual cause of right iliac fossa pain for the gynaecologist: Benign fallopian tube teratoma. J Obstet Gynaecol 18: 194-195, 1998.

13. Yoshioka T and Tanaka T: Mature solid teratoma of the fallopian tube: Case report. Eur J Obstet Gynecol Reprod Biol 89: 205-206, 2000.

14. Roncati L, Barbolini G, Ghirardini G and Rivasi F: Mature solid teratoma of the fallopian tube mimicking metastasis of endometrial adenocarcinoma: A case report. Int J Surg Pathol 18: 561-563, 2010.

15. Li S, Fang X, Chen J and Xia X: Mature cystic teratoma of the fallopian tube associated with incomplete uterine mediastinum: A case report and literature review. Oncol Lett 6: 153-155, 2013. 